\title{
Danian: Redução da latência de calda de aplicações em rede através de um escalonador $\mathbf{O}(1)$
}

\author{
Gustavo Pantuza ${ }^{1}$, Lucas A. C. Bleme ${ }^{1}$, \\ Marcos Augusto M. Vieira ${ }^{1}$, Luiz Filipe M. Vieira ${ }^{1}$ \\ ${ }^{1}$ Departamento de Ciência da Computação (DCC) \\ Universidade Federal de Minas Gerais (UFMG), Belo Horizonte, Brasil \\ \{pantuza,mmieira, lfvieira\}edcc.ufmg.br, andreyblemelegmail.com
}

\begin{abstract}
Core allocation for application threads is a problem of reasonable complexity and computacional cost inside Unix systems. Caladan scheduler is a solution aiming to reduce the cost of how threads and core are allocated in microsecond scale. The system Danian optimizes through memoization the thread picking algorithm that picks the best thread for a given core. This operation cost dropped from $O(n)$ to $O(1)$, the $C P U$ time reduced $7 \%$, the tail latency reduced $3 \%$ on Caladan Synthetic experiment and 5\% on the Netperf experiment.
\end{abstract}

Resumo. A alocação de núcleos para threads de programas é um problema complexo e de grande custo computacional dentro de ambientes Unix. O escalonador Caladan é uma solução que otimiza na escala dos microssegundos a forma como threads e núcleos são escalonados. O sistema Danian proposto, melhora o algoritmo de escolha de threads para um núcleo a ser escalonado através de memoization. O custo dessa operação foi reduzido de $O(n)$ para $O(1)$, o tempo de CPU reduzido em 7\%, a latência de calda reduziu em $3 \%$ para o experimento Synthetic e 5\% para o experimento Netperf.

\section{Introdução}

Caladan é um escalonador de CPU eficiente que consegue alcançar melhores qualidades de serviço $(Q o S)$ como latência de calda através de um agendador de threads centralizado [Fried et al. 2020]. Esse escalonador foi desenvolvido dentro do ambiente Shenango [Ousterhout et al. 2019]: um sistema para melhorar o desempenho da comunicação entre um pacote que chega a uma interface de redes até a thread da aplicação que irá computálo.

O Shenango tenta balancear a quantidade de núcleos dedicados a uma aplicação enquanto evita a Congestão de Computação: um algoritmo proposto por esse trabalho para maximizar o uso de núcleos, enquanto estiverem ociosos aguardando algum resultado de I/O, alocando-os para outras threads.

O impacto da latência de calda [Dean and Barroso 2013] no desempenho global de aplicações distribuídas em redes é um problema conhecido. Contudo, ainda em aberto para novas abordagens de mitigar ou minimizá-la em escala.

Algumas propostas tentam resolver esse problema através de transferência (offload) de carga de trabalho computacional para o núcleo de sistema operacional como por exemplo, no gancho XDP [Høiland-Jørgensen et al. 2018] da pilha TCP/IP do kernel do 
Linux. Outras abordagem transferem a carga de trabalho diretamente para interfaces de rede inteligentes [Yang et al. 2020]. Em outros casos, transfere-se o código para ambos: kernel e interface de redes [Moon et al. 2020] através de soluções como as oferecidas pelas primitivas da linguagem P4 [Bosshart et al. 2014].

Nesse contexto, o Caladan é um escalonador de threads em CPUs que visa reagir a congestionamentos na escala dos microssegundos. O presente trabalho investiga o problema da escolha de threads dentro desse escalonador. O Danian escolhe uma thread inativa que tenha sido executada recentemente no núcleo a ser escalonado. Essa abordagem reduz o tempo de carga de uma thread em um núcleo, reduz a troca de contexto e reduz a latência de calda em aplicações distribuídas dentro de centros de dados.

O Danian tem como contribuição melhorar o desempenho do Caladan na escolha de threads através do uso de memoization na estrutura de dados dos processos. A otimização implementada aumenta o uso de memória do escalador e reduz o tempo de computação do algoritmo. Nos experimentos em rede o Danian mostrou reduzir em quase $8 \%$ a latência de calda e 5\% o uso de CPU. Através de memoization o algoritmo do Danian reduz de $O(n)$ para $O(1)$ o custo para escolha de threads.

Esse documento está dividido da seguinte forma: Primeiro discute-se o estado da arte através da comparação dos trabalhos relacionados com o este trabalho. Em seguida, descreve-se o problema de escalonamento e algumas soluções já desenvolvidas para solucioná-lo. Posteriormente, apresenta-se a como o Caladan implementa seu algoritmo de escolha de threads. Depois, explica-se a solução proposta pelo Danian e como ela otimiza a escolha de threads por núcleo a ser escalonado. Em seguida, são apresentados o ambiente de experimentação e as ferramentas de avaliação. Depois, são demonstrados os resultados e análises, os potenciais trabalhos futuros e uma conclusão.

\section{Trabalhos relacionados}

O artigo RSS++ [Barbette et al. 2019] apresenta uma política de balanceamento de carga que modifica dinamicamente a indireção da busca na tabela do RSS (Receive Side Scaling). Essa política distribui a carga entre os núcleos (CPUs) de maneira ótima. O RSS++ pode ser implementado usando DPDK e executado em sistema operacional Linux tradicional em espaço de usuário (user space). Ele se mostra eficiente para fluxos que tem duração parecida. Nesses casos o RSS++ distribui a carga entre os núcleos de processamento de maneira eficiente. A proposta do presente trabalho não verifica as filas de pacotes na interface de redes. No entanto, na esfera do escalonador Caladan, implementa uma política que associa threads recentemente executadas em um dado núcleo de modo a minimizar a troca de contexto.

Algumas soluções para processamento rápido de pacotes utilizam a máquina virtual eBPF [Vieira et al. 2019] disponível no núcleo do sistema operacional Linux para fazer transferência (offload) de carga de trabalho para o núcleo do sistema operacional. Este trabalho vai na direção contrária: ao invés de transferir para o núcleo, faz-se o kernel bypass através da ferramenta DPDK [DPDK 2021].

O Caladan [Fried et al. 2020] é o escalonador objeto do presente trabalho de modo que através das otimizações para o algoritmo que associa threads a núcleos, investiga-se uma abordagem que possa reduzir a latência de aplicações em rede. 
O Shenango [Ousterhout et al. 2019] é uma solução baseada em interrupções nas interfaces e que implementa um algoritmo de detecção de congestionamento de computação. Dessa foma o Shenango realoca núcleos para processos que estejam sob congestionamento. O presente trabalho não modifica essa camada de detecção de congestionamento de computação mas se aproveita dessa realocação de núcleos.

O trabalho denominado Dune [Belay et al. 2012] investiga a possibilidade de utilizar os recursos VT-x dos processadores da Intel às quais habilitam otimizações no hardware para otimizar o desempenho de processos no sistema operacional. Para tal, o autores descrevem um módulo para o kernel do Linux que faz a interface entre os processos e os núcleos através do uso de Hypercalls. Diferentemente, o presente trabalho não depende das chamadas de sistemas de hypervisors. Utiliza-se o DPDK para implementar o kernel bypass.

Aplicações em redes que são intensivas em dados como redes sociais, buscas textuais, streaming de vídeo são normalmente distribuídas em múltiplos servidores. Tentar minimizar a latência de calda é uma tarefa complexa e que impacta a experiência dos usuários na ponta final dessas comunicações conforme demonstrado em [Dean and Barroso 2013]. O presente trabalho se baseia nesse fato para avaliar as soluções de escalonamento propostos em função da latência de calda das aplicações medidas de modo a minimizá-la.

Otimizações na esfera dos algoritmos são formas complementares de otimizar soluções [Cormen et al. 2009]. A técnica de evitar computações repetitivas na forma de memoization é um recurso comumente utilizado em soluções baseadas em programação dinâmica [Bellman 1954]. Dessa forma, o presente trabalho implementa uma solução utilizando memoization para otimizar um algoritmo que apresenta computações repetitivas para escolher a melhor thread para um dado núcleo.

\section{Escalonamento de threads}

Escalonamento de recursos em sistemas multi-thread é um problema complexo. A decisão de qual aplicação/cliente priorizar durante a tarefa de multiplexação nem sempre é justa.

O sistema Lottery [Waldspurger and Weihl 1994], propõe um mecanismo de escalonamento de recursos randomizado. Assim como em uma loteria, cada cliente recebe um número finito de bilhetes. Em cada loteria, o recurso é alocado para o cliente sorteado. Essa abordagem garante uma alocação proporcional ao número de bilhetes que um cliente possui.

Outra solução avalia e discute o problema de que a gestão de threads não é eficiente nem no espaço de kernel nem no espaço de usuário. Não exclusivamente em cada um desses ambientes. O artigo propõe e avalia o scheduler activation [Anderson et al. 1991], um contexto de execução que disponibiliza vetores de controle do kernel para o espaço de endereçamento de uma aplicação em espaço de usuário fazendo uso de eventos do kernel. Ou seja, um par entre núcleo e espaço de usuário para maximizar o escalonamento de threads.

As propostas acima não mitigam o problema de reagir em escala de microssegundos quando a latência de calda é impactada por rajadas momentâneas de requisições para 
um mesmo serviço. Para esses cenários, o escalonador do Caladan se apresenta como solução eficiente. E é nesse cenário que o Danian otimiza o Caladan para reduzir ainda mais a latência das aplicações em rede.

\section{Caladan}

Em uma máquina rodando o sistema Caladan, um núcleo é dedicado a fazer, exclusivamente, a tarefa de scheduler. Esse núcleo continuamente lê sinais de controle em uma frequência de microssegundos de modo a reagir a problemas antes mesmo que a qualidade do serviço possa degradar-se.

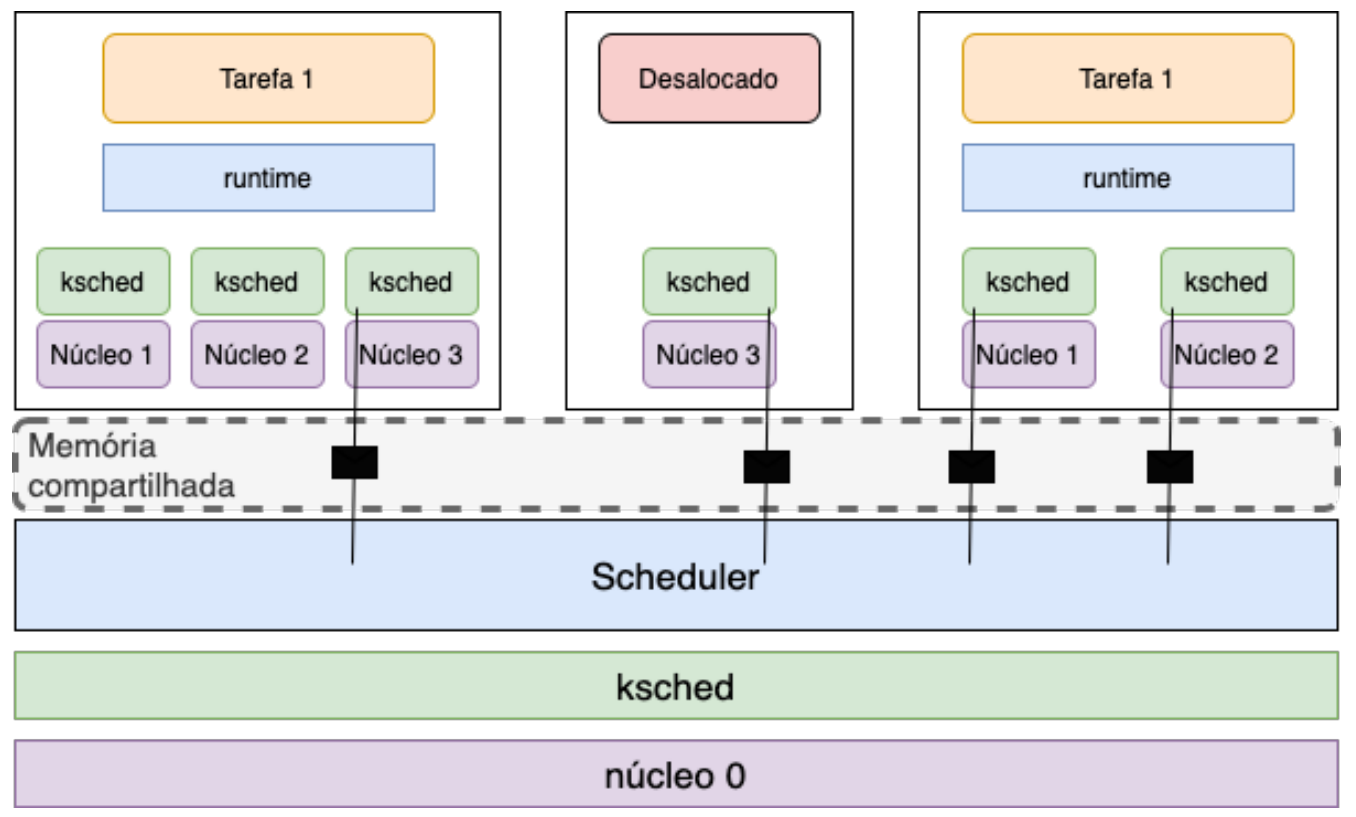

Figura 1. Imagem que apresenta a arquitetura geral do scheduler centralizado

A Figura 1 mostra a arquitetura do Caladan. O núcleo centralizado e chamado de Scheduler é responsável por ler sinais de controle dos runtimes das threads e das regiões de memória compartilhada. Assim, o Scheduler consegue ajustar a alocação de núcleos para threads que precisam ser escalonadas.

O Caladan descreve um módulo para o kernel do Linux chamado KSCHED. Em uma escala de microssegundos, esse módulo consegue fazer o agendamento de núcleos para threads. Uma instância desse módulo está associada a cada núcleo da máquina disponível para o Caladan. O agendamento é assíncrono, pois assim o agendador pode executar outras tarefas enquanto aguarda um núcleo completar uma tarefa.

O Caladan utiliza uma estrutura de dados de lista encadeada para guardar quais as threads estão em estado idle (disponíveis) dentro do escopo de um processo:

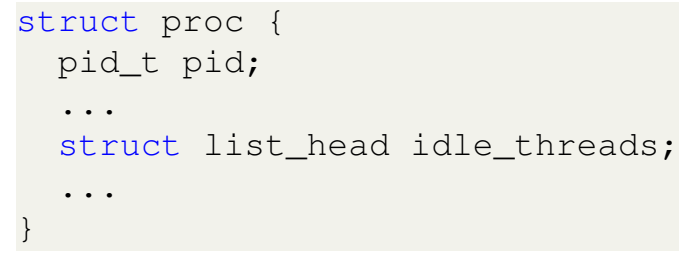

Listing 1. Lista duplamente encadeada original do Caladan 
Nessa abordagem, toda vez que o escalonador precisa escolher uma thread ele itera sobre essa lista duplamente encadeada em busca da thread disponível e que tenha sido executada recentemente no núcleo a ser utilizado. Essa solução executa em $O(n)$, onde $n$ é o número de threads executadas para um dado processo. Quando nenhuma thread em idle está disponível para ser escolhida, o algoritmo escolhe a última thread nessa lista duplamente encadeada.

O Código em linguagem $\mathrm{C}$ abaixo descreve a função sched_pick_kthread utilizada pelo Caladan para fazer a escolha da thread para um dado núcleo e processo passados como parâmetros dessa função.

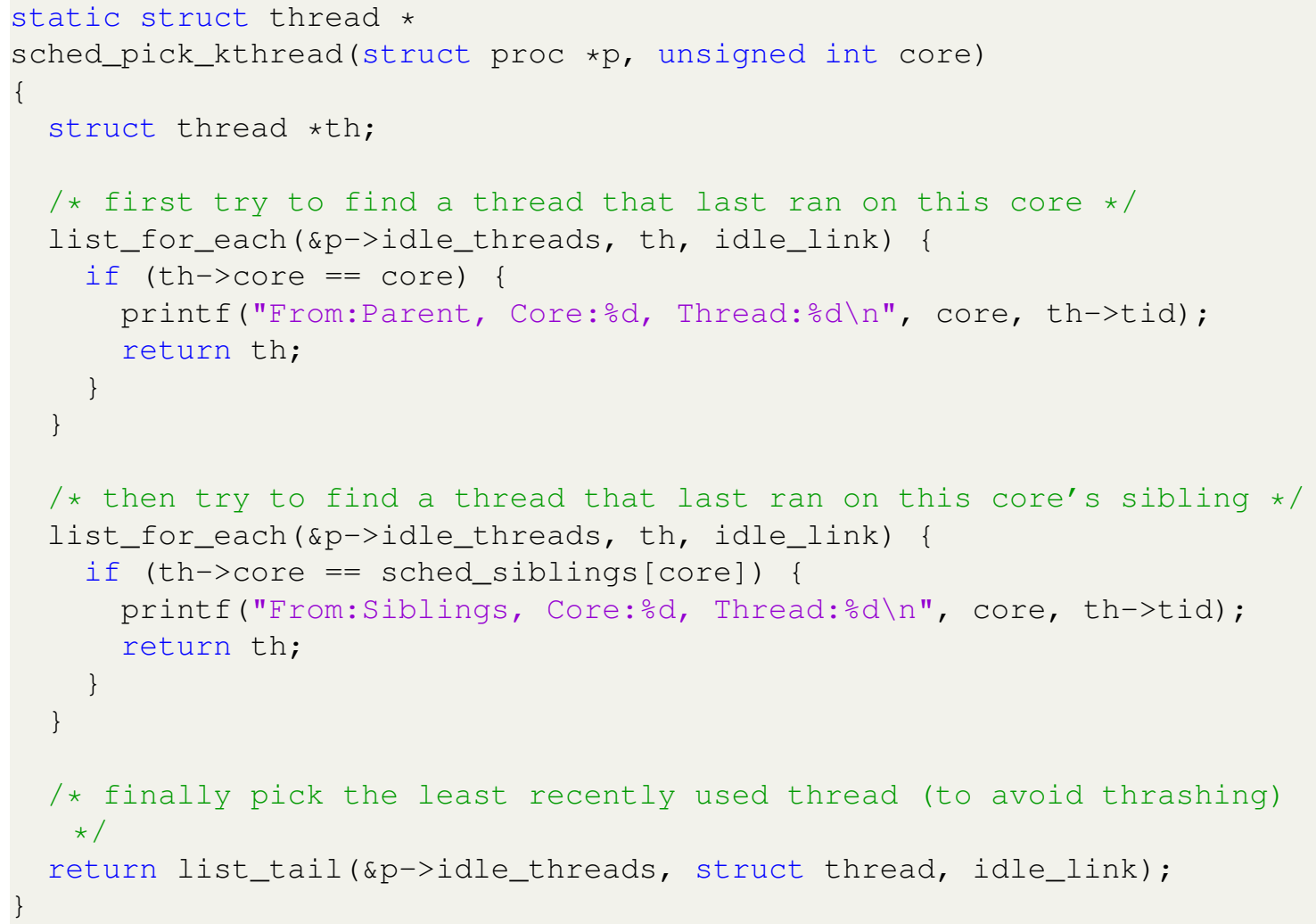

Listing 2. Função do Caladan para escolher uma thread para um núcleo

A escolha de uma thread em idle de um processo para um dado núcleo é o objeto de pesquisa do presente trabalho. A seção 5 descreve os novos algoritmos para otimizar a escolha de threads para um dado núcleo.

\section{Danian}

Para melhorar o desempenho desse algoritmo, o presente trabalho propôs o Danian: uma solução que adiciona um array de tamanho $N$ dentro da estrutura de dados de um processo. $N$ é o número de núcleos disponíveis na máquina em questão. Cada posição desse array representa um núcleo.

O Código 3 mostra a estrutura de dados adicionada ao struct proc. Esse struct representa um processo Caladan. Cada processo então passa a ter um array de memoization em que cada elemento do array é um ponteiro para uma thread, na forma de um struct thread $*$, do processo em questão. 


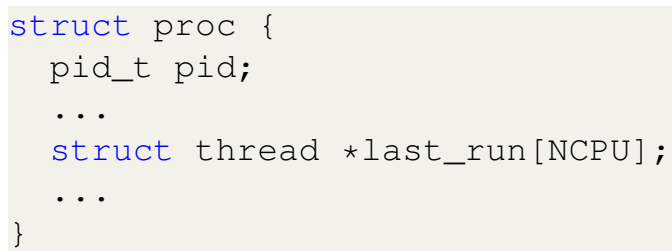

Listing 3. Estrutura de dados de memoization por processo

Todas as posições do array são inicializadas com o valor $N U L L$. Quando uma thread é escalonada para ser executada em um núcleo, atualiza-se a posição correspondente àquele núcleo para apontar para a thread em execução. Por exemplo, se a thread 5 de um dado processo é colocada para ser executada no núcleo 8 , atualiza-se a posição 8 do array last_run com o ponteiro da thread escalonada. Dessa forma, sempre atualizase no array de memoization qual a thread daquele processo rodou recentemente naquele núcleo. O índice do array é o núcleo e o conteúdo é a lembrança (memoization) da thread em questão.

O código 4 mostra a implementação da função que escolhe uma thread baseada na busca lookup dentro do array de memoization de um dado processo. Essa operação de lookup executa em $O(1)$ em seu caso médio assim como em seu pior caso. Considere-se o pior caso quando a thread não está disponível para ser escolhida, pois ainda está ativa em algum núcleo. Nesse caso, retorna-se a última thread adicionada à lista duplamente encadeada utilizada pelo Caladan originalmente conforme citado anteriormente na seção 4. Buscar a calda dessa lista encadeada é uma operação também feita em $O(1)$.

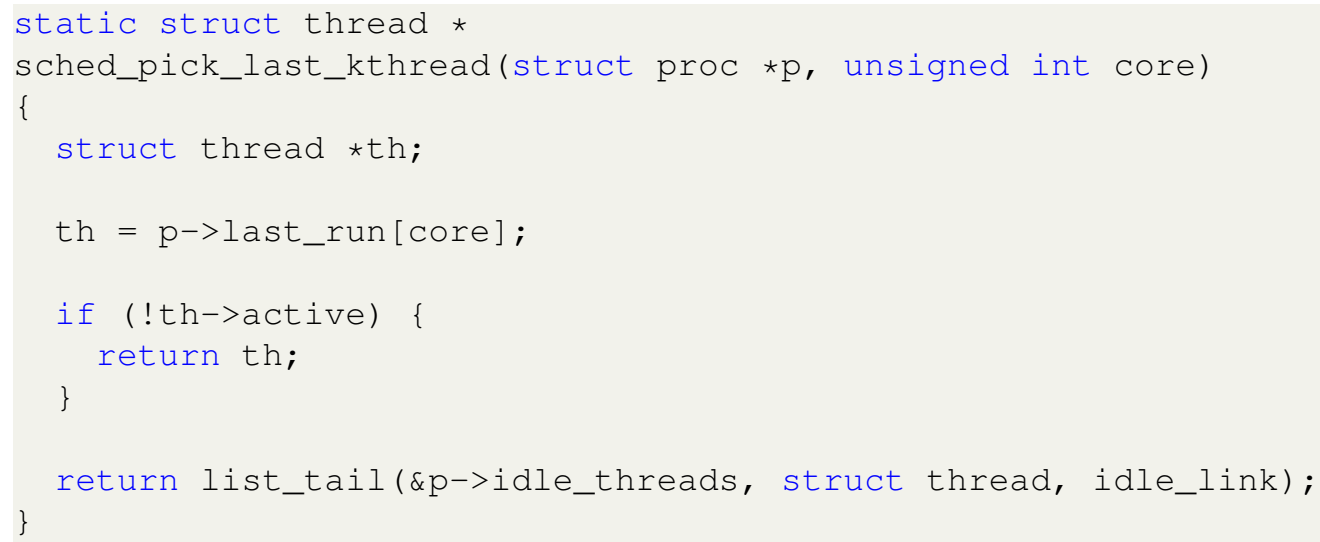

\section{Listing 4. Código que escolhe a thread para o núcleo usando memoization}

Com isso, esse array de memoization por Processo em execução permite ao escalonador encontrar as threads disponíveis de um processo que melhor se enquadram na política de escalonamento. A saber, a thread daquele processo que recentemente foi executada naquele núcleo. Quando a thread não puder ser escalonada por não estar disponível, a solução proposta pega a calda da lista encadeada de modo a evitar desperdício (trashing) por parte do escalonador e sempre retornar uma thread para o escalonador.

Esse algoritmo implementa uma política LRU (last recent used) em que escolhese a thread que mais recentemente foi executada em um dado núcleo. Essa estratégia visa reduzir a troca de contexto entre threads sendo carregadas em um núcleo. 


\section{Experimentos}

Para avaliar a solução proposta o presente trabalho executou dois experimentos: com o serviço synthetic e com o serviço netperf, ambos disponíveis no código fonte do Caladan implementados utilizando o iokernel (escalonador) mostrado na figura 1 como KSCHED. Esses programas funcionam na forma de um par de um cliente e um servidor. Ambas partes dos programas dependem do ambiente Caladan.

Os experimentos foram executados no CloudLab [Duplyakin et al. 2019] em uma topologia com duas máquinas conectadas por um switch: uma atuando como servidor e outra como cliente. A figura 2 exemplifica a topologia do experimento.

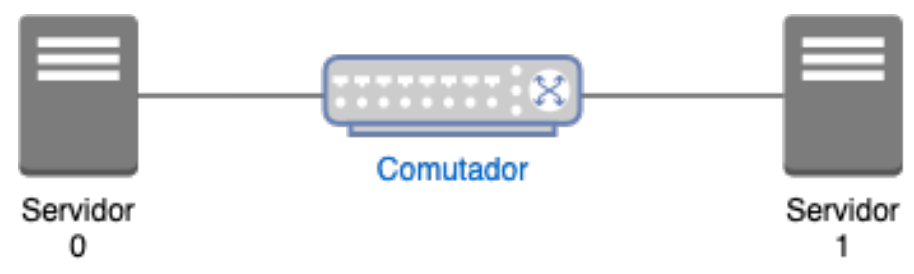

Figura 2. Topologia do experimento no CloudLab

Foram medidos percentis de latência entre o cliente e o servidor assim como tempo de execução da função que faz a escolha das threads. A latência de calda e o percentual de CPU foram computados durante os experimentos. Para o experimento netperf variou-se o número de threads utilizadas pelo cliente de modo a verificar o impacto dessa variação na latência global de uma aplicação distribuída em redes.

As máquinas servidor 0 e servidor 1 são computadores com 16 núcleos e interfaces de redes Gigabit, conforme a tabela 1

\begin{tabular}{c|c|c|c|c} 
Máquina & CPU & $\begin{array}{c}\text { Memória } \\
\text { RAM }\end{array}$ & Disco & $\begin{array}{c}\text { Interface de } \\
\text { Redes }\end{array}$ \\
\hline \multirow{2}{*}{ m510 } & 8 dual core & $64 \mathrm{~Gb}$ & & Dual-port Mellanox \\
& $\begin{array}{c}\text { Intel Xeon D-1548 } \\
\text { à } 2.0 \mathrm{GHz}\end{array}$ & $\begin{array}{c}\text { 4x 16 GB } \\
\text { DDR4 }\end{array}$ & $256 \mathrm{~GB}$ & $\begin{array}{c}\text { ConnectX } \\
\text { Portas de } 10 \mathrm{~Gb}\end{array}$
\end{tabular}

Tabela 1. Configuração das máquinas utilizadas nos experimentos

\section{Resultados}

$\mathrm{Na}$ análise comparativa entre o escalonador do Caladan nativo e o escalonador com memoization de thread por CPU, tem-se que para todas as operações dinâmicas da estrutura de dados a complexidade de tempo de execução de $O(1)$. Já para o Caladan nativo, somente a operação de inserção executa em $O(1)$. As demais operações são em $O(n)$, onde $n$ é o número de núcleos (CPUs) disponíveis na máquina. A tabela 2 apresenta a comparação entre as implementações:

Como ambas estruturas de dados dependem diretamente do número de núcleos disponíveis na máquina, elas tem complexidade de espaço em $O(n)$. Em função disso pode-se afirmar que, para otimizar o tempo de execução do algoritmo, foi necessário utilizar mais espaço em memória principal. A saber, cada processo armazena um novo 


\begin{tabular}{|l|c|c|c|c|c|c|}
\hline \multirow{2}{*}{ Programa } & \multicolumn{4}{|c|}{$\begin{array}{c}\text { Complexidade } \\
\text { de Tempo }\end{array}$} & $\begin{array}{c}\text { Complexidade } \\
\text { de Espaço }\end{array}$ \\
\cline { 2 - 6 } & Inicialização & Inserçãa & Remoção & Atualização & Busca & \\
\hline Caladan & $\mathrm{O}(\mathrm{n})$ & $\mathrm{O}(1)$ & $\mathrm{O}(\mathrm{n})$ & $\mathrm{O}(\mathrm{n})$ & $\mathrm{O}(\mathrm{n})$ & $\mathrm{O}(\mathrm{n})$ \\
\hline Danian & $\mathrm{O}(\mathrm{n})$ & $\mathrm{O}(1)$ & $\mathrm{O}(1)$ & $\mathrm{O}(1)$ & $\mathrm{O}(1)$ & $\mathrm{O}(\mathrm{n})$ \\
\hline
\end{tabular}

Tabela 2. Tabela comparativa de complexidades de tempo e espaço das implementações do Caladan e do Caladan otimizado com memoization

array de tamanho $n$, onde $n$ representa o número de núcleos disponíveis para o Caladan. Nesse caso, se tivermos 30 processos, nas máquinas do experimento (com 16 núcleos), teremos um acréscimo de alocação de $30 * n *$ sizeof (struct thread $*$ ), onde 30 representa o número de processos, $n$ o número de núcleos e sizeof (structthread $*$ ) representa o espaço necessário pelo código em $\mathrm{C}$ para alocar um ponteiro de uma estrutura de dados thread.

\section{Tempo de execução da função de escolha de threads em (ns)}

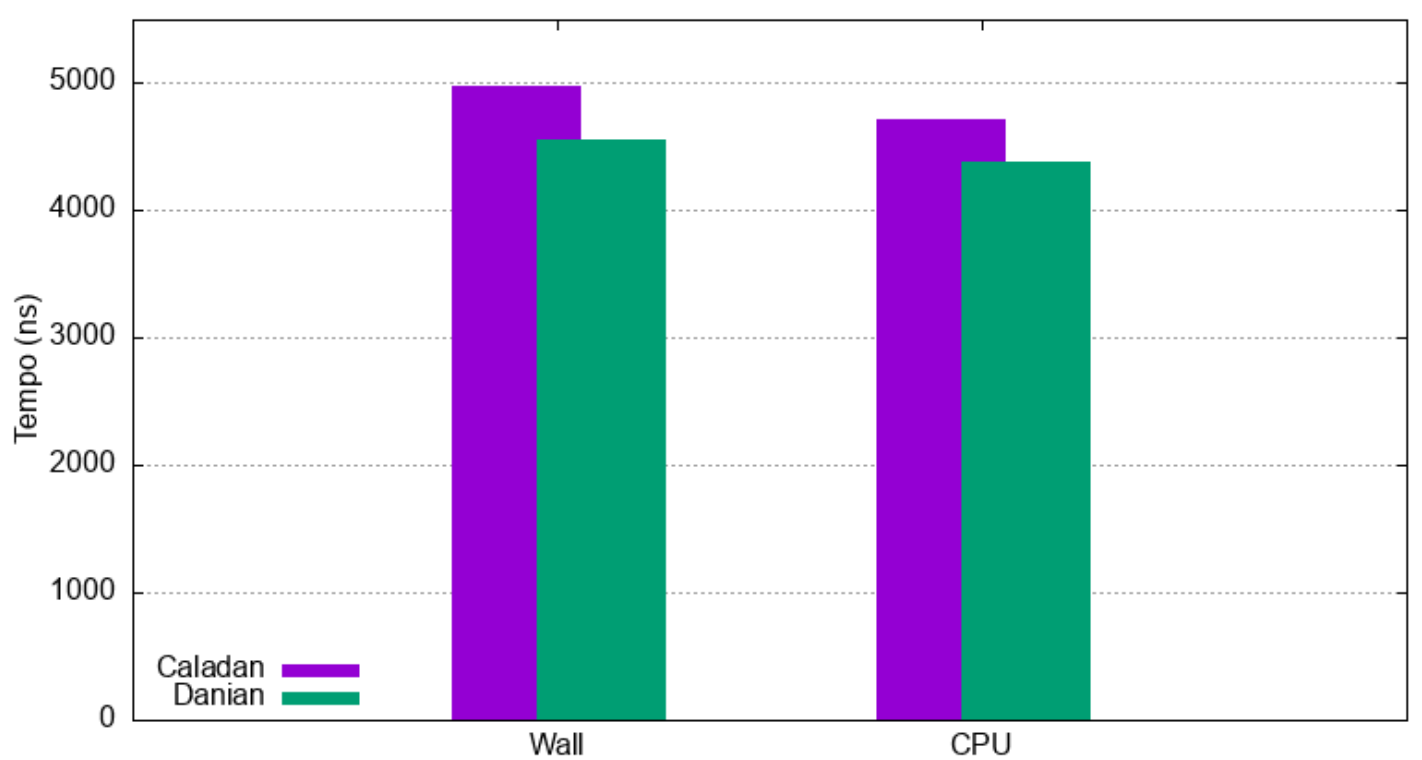

Figura 3. Tempos de CPU e Wall contabilizados para o Caladan e Danian

Notou-se, para o escopo do experimento com o programa synthetic uma redução no tempo de execução com a função sched_pick_last_kthread comparada à sched_pick_kthread. Foram contabilizados os tempos de CPU e Wall. Verificou-se uma redução de aproximadamente $7 \%$ no tempo de execução. A figura 3 mostra o gráficos dos tempos contabilizados em cada versão do escalonador.

Durante o experimento avaliou-se comportamento da latência de calda do serviço synthetic entre o cliente e o servidor. A figura 4 apresenta o comparativo das latências medidas em ambos os programas para o mesmo experimento. À cada número de coleta o programa synthetic aumenta linearmente o volume de dados transferidos. A implementação proposta mostrou uma redução de $3 \%$ no percentil 99 da latência durante a execução do 


\section{Comparação do percentil 99 da latência durante execução do Synthetic}

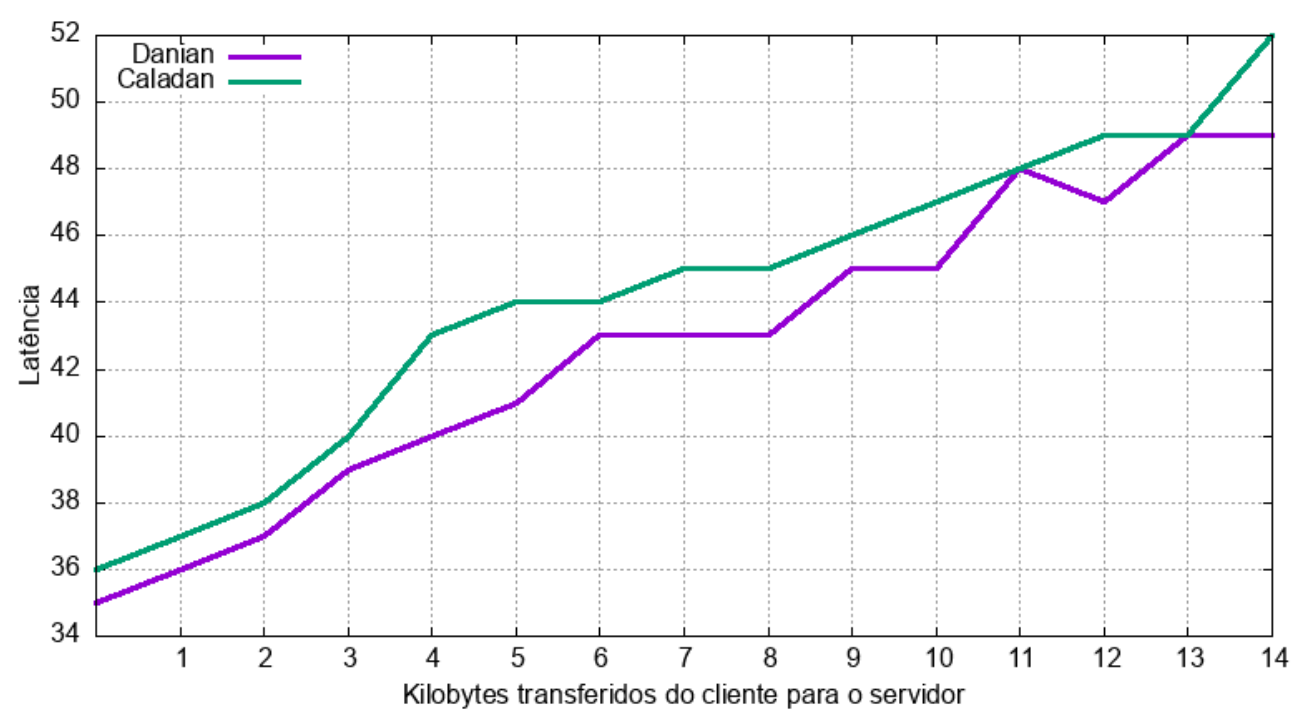

Figura 4. Latência de calda no percentil 99 do experimento Synthetic

synthetic.

Para o experimento netperf foram executadas diversas baterias de experimentação onde variou-se o número de threads na máquina cliente de 2 threads até 12 threads, aumentando de duas em duas threads.

A figura 5 mostra como à medida que o número de threads aumenta, a latência do experimento reduz. Esse comportamento ocorreu em ambos os casos: Caladan baseline e com a otimização de memoization. No entanto, o último manteve uma latência média menor em todos os cenários do experimento.

\section{Latências médias em função do número de threads}

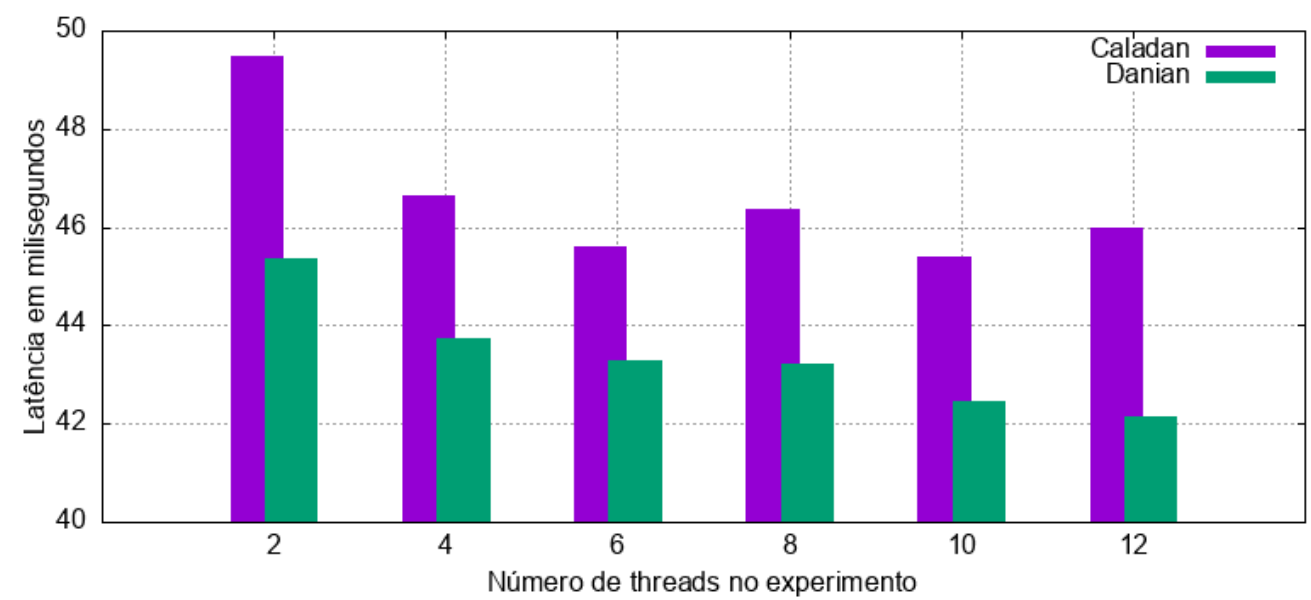

Figura 5. Latência média em função do número de threads no cliente

Durante o mesmo experimento variando o número de threads analisou-se o per- 
centual de uso de CPU. A figura 6 mostra a dispersão dos usos de CPU computados para cada volume de pacotes enviados do cliente para o servidor.

\section{Dispersão e médias dos percentuais de uso de CPU}

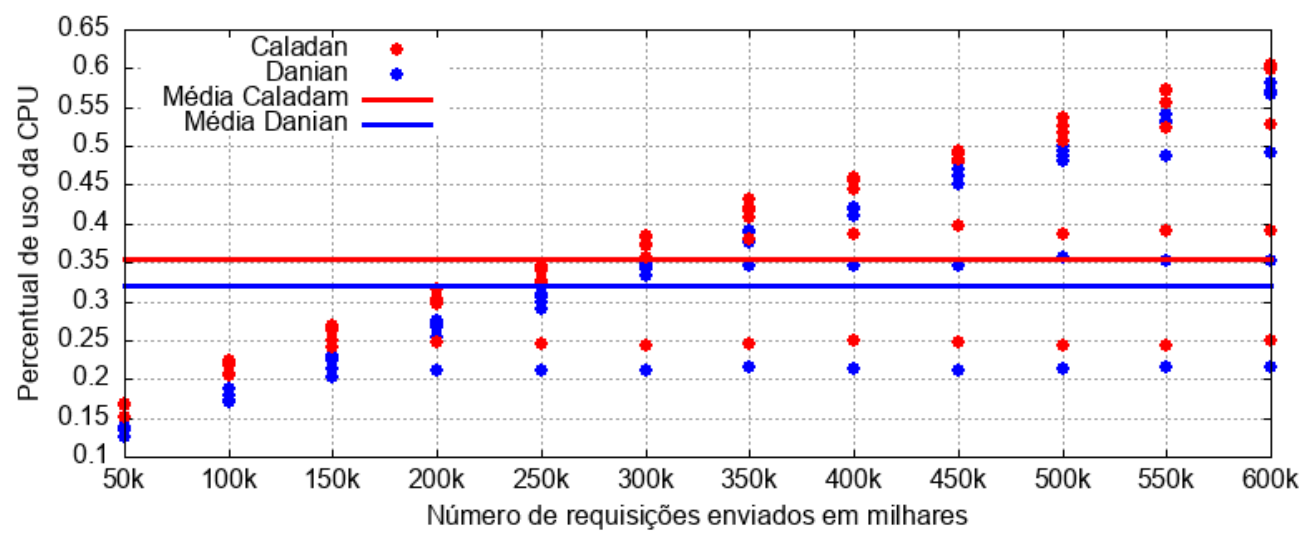

Figura 6. Médias de percentuais de CPU utilizada durante o experimento

Como pode ser notado nas linhas horizontais desse gráfico mostrando as médias globais dos percentuais de CPU, o algoritmo de otimizado com memoization reduz em quase $5 \%$ o uso de CPU pelo programa netperf.

A latência de calda do experimento netperf foi medida no percentil 90 comparando as latências entre o Caladan baseline e o otimizado com memoization. A figura 7 mostra esse resultado e é possível observar a redução na latência à medida que o número de pacotes enviados por coleta do experimento era executado.

\section{Percentil 90 da latência durante execução do NetPerf à medida que o número de requisições cresce}

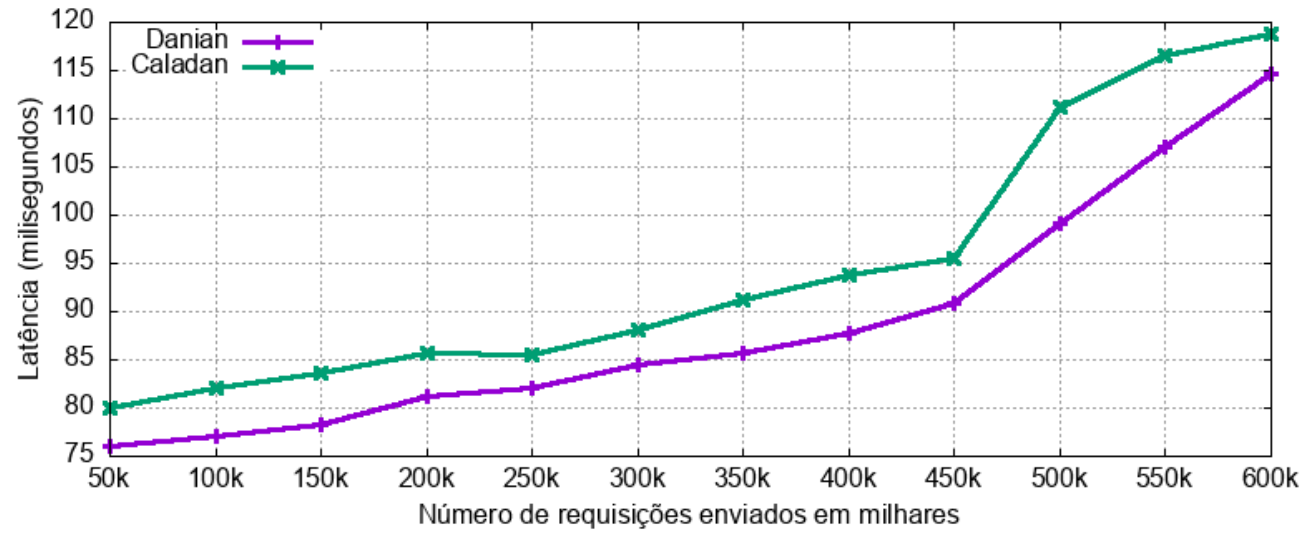

Figura 7. Latência de calda medida no experimento netperf

Através desses experimento foi possível comparar e medir o desempenho da função apresentada no código 4 ao qual a função sched_pick_last_khread() faz uso do array de memoization por processo para lembrar e buscar qual foi a última thread executada para um dado núcleo em uma política de LRU. 
Durante o experimento Netperf, observou-se uma melhoria na vazão de pacotes à medida que o número de threads foram aumentadas. Uma melhoria média de $3 \%$ pode ser verificada na figura 8 comparando a solução Danian ao Caladan. Note que as curvas de vazão de requisições convergem ao final do experimento (12 threads) para 600 mil. Esse é o limite de I/O do sistema experimentado.

\section{Requisições por segundo durante o NetPerf à medida que o número de threads aumenta}

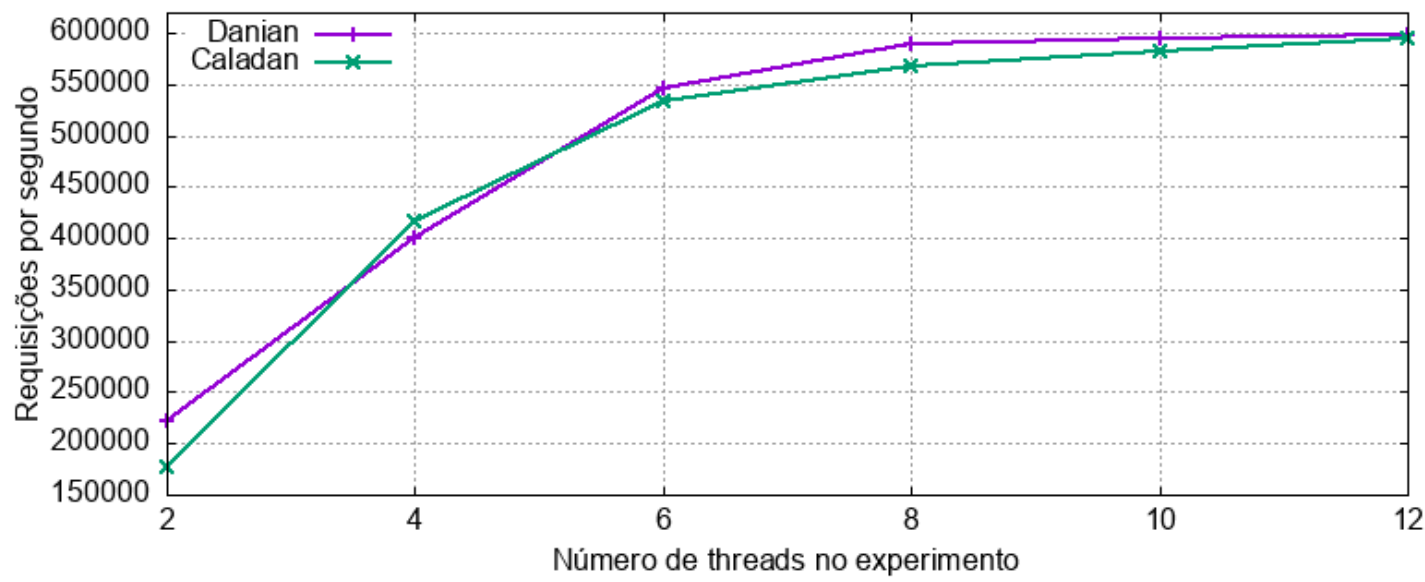

Figura 8. Requisições por segundo em funções do número de threads

\section{Trabalhos Futuros}

Em um cenário com múltiplas tarefas sendo acomodadas pelo escalonador Caladan, a latência e utilização dos ciclos de CPU pode se comportar de maneira diferente em relação ao experimento apresentado neste trabalho. Tendo por exemplo uma tarefa que processa pacotes provenientes de um banco de dados memcached, e outra tarefa com um garbage collector sendo executada em background, o escalonador Caladan pode realizar ainda mais operações de escolha de thread.

Desta maneira, a presente implementação proposta com memoization no mecanismo de escolha entre threads pode se mostrar ainda mais eficiente em cenários onde há interferência entre tarefas, se apresentando assim como interessante oportunidade de análise futura.

O Caladan tem potencial de ser utilizado para implementar uma solução em SDN, em que pode-se separar o plano de dados e o plano de controle [Pantuza et al. 2014]. Em especial para um plano de dados eficiente e de baixa latência.

O Caladan pode ser utilizado como escalonador para aplicações utilizando lthreads [Rushing's 2021]. Seria possível criar funções de rede (NFVs) eficientes e fazer experimentos comparativos verificando o desempenho dessas funções.

Uma avaliação detalhada do algoritmo em diversos outros cenários e programas seriam interessantes para estressar os limites da solução proposta, que, utilizando uma estrutura de dados com complexidade de tempo constante $O(1)$ em lookup otimiza a utilização de CPU e reduzir latência no processamento de pacotes. 


\section{Conclusão}

O presente trabalho melhora o desempenho do escalonador de CPU Caladan na forma como ele escolhe threads a serem alocadas em um dado núcleo do computador. A solução proposta reduz de $O(n)$ para $O(1)$ a complexidade de tempo de execução do algoritmo que escolhe uma thread de um processo para ser executada em um núcleo. Todas as demais operações dinâmicas utilizadas na estrutura de dados proposta também foram reduzidas a $O(1)$. Em contrapartida, aumentou-se o espaço de memória alocado por processo dentro do escalonador.

Através do uso de memoization com a política LRU, o Danian reduziu em $7 \%$ o tempo de CPU e 3\% a latência de calda medida através do percentil 99 do experimento Synthetic enquanto manteve a capacidade do escalonador de alocar novos núcleos de processamento em microssegundos.

Além disso, para um experimento de desempenho da rede (netperf) reduziu-se a latência média em 5\% e o percentual de uso de CPU em 15\%. Uma análise do comportamento da latência mostra que à medida que o número de threads aumenta, a solução proposta reduz a latência global de round trip (RTT) da aplicação.

O Shenango e o Caladan são o estado da arte em eficiência na maximização do uso de CPU em um computador rodando aplicações multi-thread. O presente trabalho é uma contribuição para tornar essas ferramentas mais eficientes e capazes de processar pacotes de aplicações em rede que fazem uso intensivo de dados e exigem cada vez mais baixa latência.

\section{Referências}

Anderson, T. E., Bershad, B. N., Lazowska, E. D., and Levy, H. M. (1991). Scheduler activations: Effective kernel support for the user-level management of parallelism. SIGOPS Oper. Syst. Rev., 25(5):95-109.

Barbette, T., Katsikas, G. P., Maguire, G. Q., and Kostić, D. (2019). Rss++: Load and state-aware receive side scaling. In Proceedings of the 15th International Conference on Emerging Networking Experiments And Technologies, CoNEXT '19, page 318-333, New York, NY, USA. Association for Computing Machinery.

Belay, A., Bittau, A., Mashtizadeh, A., Terei, D., Mazières, D., and Kozyrakis, C. (2012). Dune: Safe user-level access to privileged CPU features. In 10th USENIX Symposium on Operating Systems Design and Implementation (OSDI 12), pages 335-348, Hollywood, CA. USENIX Association.

Bellman, R. (1954). The theory of dynamic programming. Bulletin of the American Mathematical Society, 60(6):503 - 515.

Bosshart, P., Daly, D., Gibb, G., Izzard, M., McKeown, N., Rexford, J., Schlesinger, C., Talayco, D., Vahdat, A., Varghese, G., and Walker, D. (2014). P4: Programming protocol-independent packet processors. SIGCOMM Comput. Commun. Rev., 44(3):87-95.

Cormen, T. H., Leiserson, C. E., Rivest, R. L., and Stein, C. (2009). Introduction to Algorithms, Third Edition. The MIT Press, 3rd edition. 
Dean, J. and Barroso, L. A. (2013). The tail at scale. Communications of the ACM, $56: 74-80$.

DPDK (2021 (acessado em Março de 2021)). Data Plane Development Kit.

Duplyakin, D., Ricci, R., Maricq, A., Wong, G., Duerig, J., Eide, E., Stoller, L., Hibler, M., Johnson, D., Webb, K., Akella, A., Wang, K., Ricart, G., Landweber, L., Elliott, C., Zink, M., Cecchet, E., Kar, S., and Mishra, P. (2019). The design and operation of cloudlab. In 2019 USENIX Annual Technical Conference (USENIX ATC 19), pages 1-14, Renton, WA. USENIX Association.

Fried, J., Ruan, Z., Ousterhout, A., and Belay, A. (2020). Caladan: Mitigating interference at microsecond timescales. In 14th USENIX Symposium on Operating Systems Design and Implementation (OSDI 20), pages 281-297. USENIX Association.

Høiland-Jørgensen, T., Brouer, J. D., Borkmann, D., Fastabend, J., Herbert, T., Ahern, D., and Miller, D. (2018). The express data path: Fast programmable packet processing in the operating system kernel. In Proceedings of the 14th International Conference on Emerging Networking EXperiments and Technologies, CoNEXT'18, page 54-66, New York, NY, USA. Association for Computing Machinery.

Moon, Y., Lee, S., Jamshed, M. A., and Park, K. (2020). Acceltcp: Accelerating network applications with stateful TCP offloading. In 17th USENIX Symposium on Networked Systems Design and Implementation (NSDI 20), pages 77-92, Santa Clara, CA. USENIX Association.

Ousterhout, A., Fried, J., Behrens, J., Belay, A., and Balakrishnan, H. (2019). Shenango: Achieving high CPU efficiency for latency-sensitive datacenter workloads. In 16th USENIX Symposium on Networked Systems Design and Implementation (NSDI 19), pages 361-378, Boston, MA. USENIX Association.

Pantuza, G., Sampaio, F., Vieira, L. F. M., Guedes, D., and Vieira, M. A. M. (2014). Network management through graphs in software defined networks. In 10th International Conference on Network and Service Management (CNSM) and Workshop, pages 400-405.

Rushing's, S. (2021 (acessado em Março de 2021)). Coroutine library threads.

Vieira, M. A. M., Castanho, M. S., Pacífico, R. D. G., Santos, E. R. S., Câmara Júnior, E. P. M., and Vieira, L. F. M. (2019). Processamento Rápido de Pacotes com eBPF e XDP. In Minicursos do XXXVII Simpósio Brasileiro de Redes de Computadores e Sistemas Distribuidos (SBRC), Porto Alegre, RS, Brasil. SBC.

Waldspurger, C. A. and Weihl, W. E. (1994). Lottery scheduling: Flexible proportionalshare resource management. In Proceedings of the 1st USENIX Conference on Operating Systems Design and Implementation, OSDI '94, page 1-es, USA. USENIX Association.

Yang, X., Eggert, L., Ott, J., Uhlig, S., Sun, Z., and Antichi, G. (2020). Making quic quicker with nic offload. In Proceedings of the Workshop on the Evolution, Performance, and Interoperability of QUIC, EPIQ '20, page 21-27, New York, NY, USA. Association for Computing Machinery. 